\title{
Model-based registration for assessment of spinal deformities in idiopathic scoliosis
}

\author{
Daniel Forsberg, Claes Lundström, Mats Andersson and Hans Knutsson
}

\section{Linköping University Post Print}

\section{Tweet}

N.B.: When citing this work, cite the original article.

Original Publication:

Daniel Forsberg, Claes Lundström, Mats Andersson and Hans Knutsson, Model-based registration for assessment of spinal deformities in idiopathic scoliosis, 2014, Physics in Medicine and Biology, 59(2), 311-326.

http://dx.doi.org/10.1088/0031-9155/59/2/311

Copyright: IOP Publishing: Hybrid Open Access http://www.iop.org/

Postprint available at: Linköping University Electronic Press http://urn.kb.se/resolve?urn=urn:nbn:se:liu:diva-91233 


\title{
Model-based registration for assessment of spinal deformities in idiopathic scoliosis
}

\author{
Daniel Forsberg ${ }^{1,2,3}$, Claes Lundström ${ }^{2,3}$, Mats Andersson ${ }^{1,2}$ \\ and Hans Knutsson ${ }^{1,2}$ \\ ${ }^{1}$ Department of Biomedical Engineering, Linköping University, Sweden \\ 2 Center for Medical Image Science and Visualization (CMIV), Linköping \\ University, Sweden \\ 3 Sectra, Linköping, Sweden \\ E-mail: daniel.forsberg@liu.se
}

\begin{abstract}
Detailed analysis of spinal deformity is important within orthopaedic healthcare, in particular for assessment of idiopathic scoliosis. This paper addresses this challenge by proposing an image analysis method, capable of providing a full $3 \mathrm{D}$ spine characterization. The proposed method is based on the registration of a highly detailed spine model to image data from computed tomography. The registration process provides an accurate segmentation of each individual vertebra and the ability to derive various measures describing the spinal deformity. The derived measures are estimated from landmarks attached to the spine model and transferred to the patient data according to the registration result. Evaluation of the method provides an average point-to-surface error of $0.9 \mathrm{~mm} \pm 0.9$ (comparing segmentations), and an average target registration error of $2.3 \mathrm{~mm} \pm 1.7$ (comparing landmarks). Comparing automatic and manual measurements of axial vertebral rotation provides a mean absolute difference of $2.5^{\circ} \pm 1.8$, which is on par with other computerized methods for assessing axial vertebral rotation. A significant advantage of our method, compared to other computerized methods for rotational measurements, is that it does not rely on vertebral symmetry for computing the rotational measures. The proposed method is fully automatic and computationally efficient, only requiring three to four minutes to process an entire image volume covering vertebrae L5 to T1. Given the use of landmarks, the method can be readily adapted to estimate other measures describing a spinal deformity by changing the set of employed landmarks. In addition, the method has the potential to be utilized for accurate segmentations of the vertebrae in routine computed tomography examinations, given the relatively low point-to-surface error.
\end{abstract}

\section{Introduction}

Scoliosis is a disease affecting the human spine, typically characterized by introducing an abnormal lateral curvature of the spine, as observed in the coronal plane. The disease can be congenital or have neuromuscular causes, however, in most cases the cause of scoliosis is unknown, i.e. idiopathic scoliosis. Idiopathic scoliosis is usually detected during a standard physical examination, employing Adam's forward bend test. The routine procedure for assessing the severity of idiopathic scoliosis is to use a two-dimensional (2D) anterior-posterior radiograph, where the Cobb angle is measured (Cobb 1948). The Cobb angle is defined as the angle between the endplates of the end vertebrae, defining the scoliotic curvature. There exist other manual 
measurement methods for assessing coronal and also sagittal curvature of the spine, but the Cobb angle is commonly considered as gold standard. A recent review of various methods for both coronal and sagittal evaluation of spinal curvature is found in the work by Vrtovec, Pernuš and Likar (2009).

The Cobb angle plays a significant role for disease monitoring and when selecting the appropriate treatment (bracing or surgery), even though it is well-established that the Cobb angle is insufficient both to describe and to properly quantify the deformity in scoliosis. This is due to the three-dimensional (3D) nature of a scoliotic curvature, which may include a displacement, a rotation and/or a deformation of each vertebra. Another limitation of the Cobb angle is its observer-variability. Hence, other methods for quantifying the degree of scoliosis are called for, methods which can better assess the full 3D deformity of the spine in individual patients. This has been the aim of our work and we argue that our proposed method is suitable for this purpose.

Recent research has proposed axial vertebral rotation (AVR) as relevant for understanding underlying causes and processes of idiopathic scoliosis, but also for deciding upon treatment and monitoring the progression of the disease (Skalli et al. 1995, Kuklo et al. 2005, Heidari et al. 2006). Reviews regarding manual methods for measuring AVR are found in (Lam et al. 2008, Vrtovec, Pernuš and Likar 2009). This focus on AVR measurements has spawned an interest in creating computerized methods for measuring the AVR, since manual methods often suffer from being time-consuming, complex and related with a relatively high intra- and inter-observer variability.

Rogers et al. (2002) presented a method for measurements of inter-vertebral rotation in the lumbar spine, based upon image registration, applicable to both computed tomography $(\mathrm{CT})$ and magnetic resonance imaging (MRI). Adam and Askin (2006) developed a semi-automatic method for measuring the vertebral rotation based upon maximizing the symmetry ratio. Due to the applied threshold, their method is limited to CT images. Another method limited to CT images was presented by Kouwenhoven et al. (2006). Here the AVR was measured based upon the centroids of the vertebral bodies and the spinal canal. Vrtovec (2008) presented a modalityindependent method for determining the vertebral position and rotation in 3D. The method utilizes symmetry estimates to find the position and the rotation of each vertebra.

The methods of Rogers et al. (2002), Adam and Askin (2006) and Kouwenhoven et al. (2006) are limited in measurement accuracy, since they only use 2D axial images when estimating the rotation and are, thus, of limited interest, whereas the method in (Vrtovec 2008) performs the measurements in 3D. However, all four methods require more or less manual interaction, ranging from selecting which axial image to measure on to providing initial centrepoints for each vertebra, and are therefore still likely to cause an intra- and inter-observer variability. For instance, it was shown in (Vrtovec et al. 2010) that the method of Vrtovec (2008) only yielded a sligthly better inter-observer variability than the manual method by Aaro and Dahlborn (1981). In (Forsberg et al. 2013) we attempted to overcome this by presenting a fully automatic method for measuring AVR, albeit limited to CT data. A common limitation of all referenced methods for computerized AVR measurements, is their dependency on vertebral symmetry for estimating the vertebral rotation, and, as is well-established, the deformation of the vertebral body itself is an important aspect of the process related to idiopathic scoliosis (Villemure et al. 2001, Hawes and O'Brien 2006).

Another approach for assessing the scoliotic curvature, which is not limited to 
measuring only the AVR but can measure both position and rotation of each vertebra, is to use stereo-radiography and reconstruction techniques for 3D modelling of the vertebrae. Brown et al. (1976) introduced this approach and recent work is mostly related to the commercially available EOS ${ }^{\circledR}$ system (Dubousset et al. 2005). This approach is typically considered as favourable over methods based upon CT data due to the lower radiation dose associated with stereo-radiography and since CT data is acquired with the patient in supine position instead of in standing position. Disadvantages associated with this technique are long computations times and the need for manual interaction, although recent work claim to have found a suitable compromise between the amount of user interaction and the required processing time (Moura et al. 2011).

The purpose of this paper is to propose a method, capable of automatically segmenting the vertebrae and estimating various measures that describe a spinal deformity, in this paper exemplified by, but not limited to, the position and the rotation of the vertebrae in the spine. The proposed method differs from previously presented methods for AVR measurements by not being dependent on vertebral symmetry for measuring the rotation. Compared to stereo-radiography and 3D reconstructions based upon the $\operatorname{EOS}^{\circledR}$ system, it has the benefits of not requiring a dedicated imaging modality and requiring minimal user interaction. The method was developed to be used with CT data, although we believe that adapting the pre-processing is likely to make it applicable to MRI data as well.

The method is based on the idea of attaching landmarks to a model, registering the model to patient data, and thereafter transferring the landmarks of the deformed model to the patient data. The deformed model provides an accurate segmentation of the vertebrae and the transferred landmarks are utilized for measuring the position and the rotation of each vertebra. Herein resides one of the key components of the method. Adapting the set of used landmarks, other measures describing the spinal deformity, than the ones described in this paper, can readily be derived.

\section{Method overview}

The method relies on the following components; pre-processing, image registration and transfer of landmarks. In the pre-processing step, an initial pose (position and rotation) of each vertebra is estimated. This step is applied both to the spine model and to the patient data. Thereafter, each vertebra of the model is registered to the vertebrae of the patient. This is achieved by extracting a sub-volume for each vertebra from the data sets, i.e. from both the patient and the model data. The sub-volumes contain the vertebrae to be registered and any surrounding vertebrae included in the extracted sub-volumes. The sub-volumes are registered with first an affine registration, to compensate for overall differences due to translation, rotation and scale, and then with a deformable registration. The estimated transformations are composed and used to transfer the landmarks of the spine model to the spine of the patient. A graphical overview of the method is provided in figure 1.

\subsection{Spine model}

In this work, we have utilized a voxel phantom known as XCAT, developed by Segars et al. (2010), to derive our spine model. The XCAT phantom is a highly detailed phantom for both the male and the female anatomy, using non-uniform rational B- 


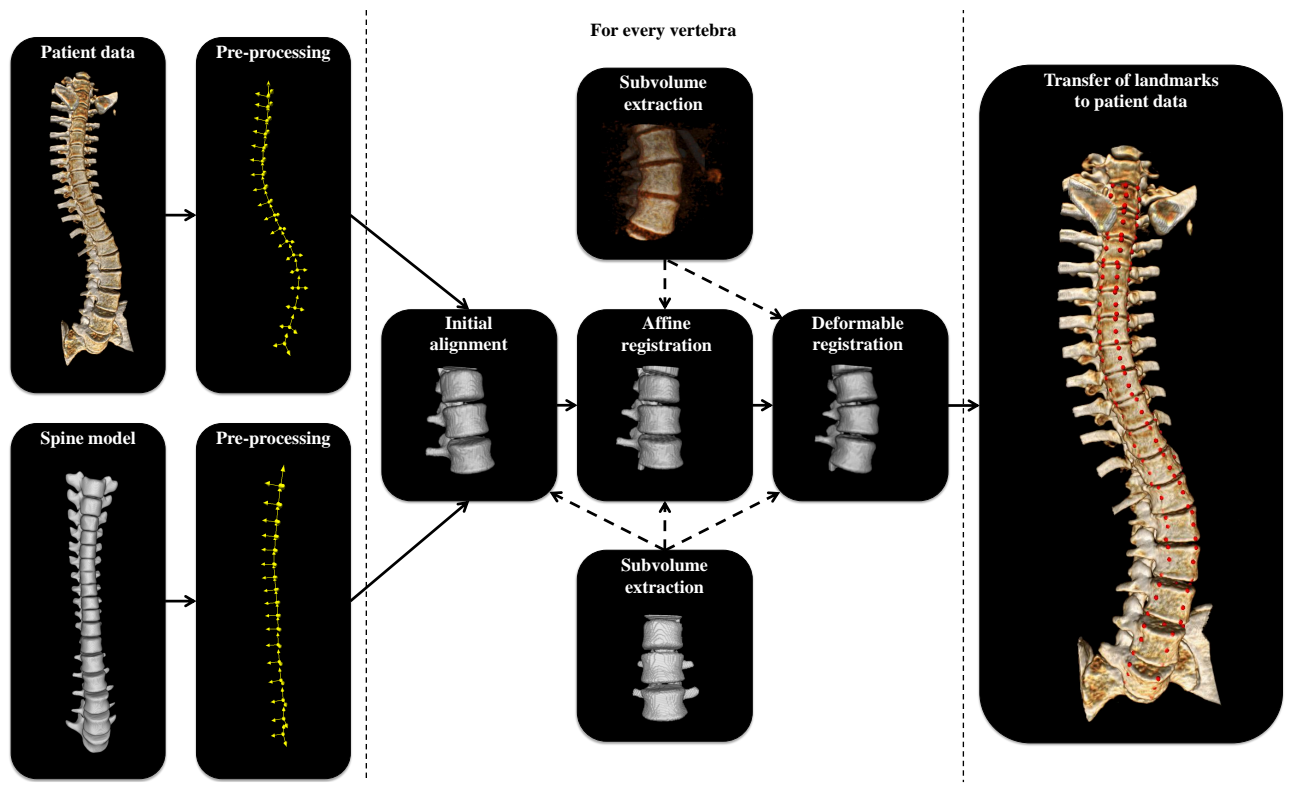

Figure 1. Schematic overview of the proposed method; pre-processing, image registration and the final transfer of the landmarks to the patient data. Note that the extracted sub-volumes from the model also contained the surrounding vertebrae. This was crucial in order to ensure that the endplates of the model vertebra of interest would not be matched to the endplates of the inferior or the superior vertebrae in the extracted sub-volume from the patient, i.e. the surrounding vertebrae served as an overlap constraint in our vertebra by vertebra registration.

splines (NURBS) for defining the anatomy of the different organs, which are initially based upon the Visible Male and Female anatomical datasets from the National Library of Medicine. The phantom is typically used for simulation studies for CT, positron emission tomography and single-photon emission computed tomography, but here we have used it to create a voxel model of all thoracic and lumbar vertebrae. The software accompanying the phantom allows the user to change a number of parameters, ranging from overall patient size and orientation to the inclusion of heart and/or respiratory motions. In this work, the female anatomy was used, since all analysed patients were female. Note that the employed model is considered to represent the normal anatomy of a spine, i.e. no scoliotic deformity was present in the model, and with a size of the spine corresponding to the 50th percentile of a female person. See (Segars et al. 2010) for further details about the XCAT phantom.

\subsection{Landmarks}

The choice of landmarks decides which measures that can be estimated and how the measures are defined. In this work, we decided to use landmarks suitable for estimating the position and the rotation of each vertebra. Note that the method is not limited to the landmarks or the measures suggested here. The landmarks were manually placed in the mid-axial, the mid-sagittal and the mid-coronal images of each model vertebra according to the scheme depicted in figure 2. The selection of these landmarks were 


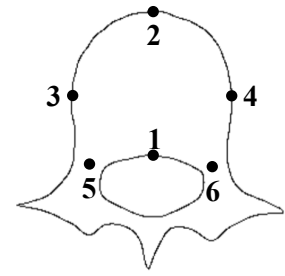

(a)

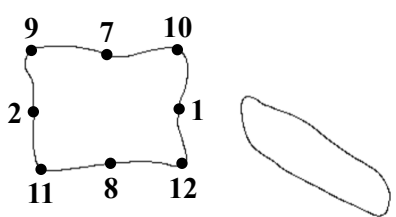

(b)

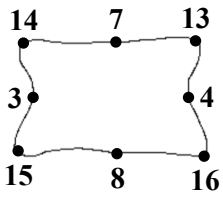

(c)

Figure 2. The following 16 landmarks were placed in the mid-axial (a), the mid-sagittal (b) and the mid-coronal (c) images of each vertebra. Landmarks 1-6 define the axial plane of the vertebra, landmarks 1-2 and 7-11 the sagittal plane and landmarks 3-4, 7-8 and 13-16 the coronal plane.

inspired by Štern et al. (2011) and the fact that they are relatively easy to place, given an orthogonal multi-planar reconstruction (MPR) viewer, and are useful for a robust estimation of the rotation matrix $\mathbf{R}$ of each vertebra. The rotation matrix $\mathbf{R}$ of each vertebra can be determined by minimizing

$$
\mathbf{R}_{0}=\underset{\mathbf{R}}{\arg \min } \epsilon^{2}(\mathbf{R})=\sum_{k=1}^{3} \sum_{l \in \Omega_{k}}\left[\left(\mathbf{R} \mathbf{e}_{k}\right)^{T} \mathbf{x}_{l}\right]^{2},
$$

where $\mathbf{e}_{1}=\left[\begin{array}{lll}1 & 0 & 0\end{array}\right]^{T}, \mathbf{e}_{2}=\left[\begin{array}{lll}0 & 1 & 0\end{array}\right]^{T}, \mathbf{e}_{3}=\left[\begin{array}{lll}0 & 0 & 1\end{array}\right]^{T}$ and $\mathbf{x}_{l}$ is a coordinate vector of the landmarks, defining the different orthogonal planes as given by the different sets $\Omega_{k}$. The optimal $\mathbf{R}_{0}$ is easily found by using a quaternion representation of $\mathbf{R}$ and by solving a non-linear least squares problem.

\subsection{Pre-processing}

To improve the initial starting point for the registration, which is necessary due to the potentially large deformation of the spine caused by the scoliosis, a pre-processing step was added to estimate an initial pose (position and rotation) of each vertebra. This step consists of the following four components; extraction of the spinal canal centreline, disc detection, vertebra centrepoint estimation and vertebra rotation estimation, which is the same method as used in our previous work (Forsberg et al. 2013), hence, details of the pre-processing can be found therein. A graphical overview of the pre-processing is provided in figure 3. Note that although the initial starting point for the registration, as estimated from the pre-processing, is based upon the symmetry of vertebral bodies, the end results obtained from the subsequent registration process do not rely on symmetric vertebral bodies.

The data was further pre-processed by applying a threshold at 100 Hounsfield units, to remove the influence of soft tissues during the registration process, and by applying a valley-emphasis, as described by Kim and Kim (2009) in order to increase the separability of surrounding vertebrae. A valley-emphasized image is created as

$$
\begin{aligned}
& (I \cdot B)(X)=(I \oplus B) \ominus B, \\
& V(X) \quad=(I \cdot B)-I, \\
& I_{V}(X) \quad=(I-V),
\end{aligned}
$$




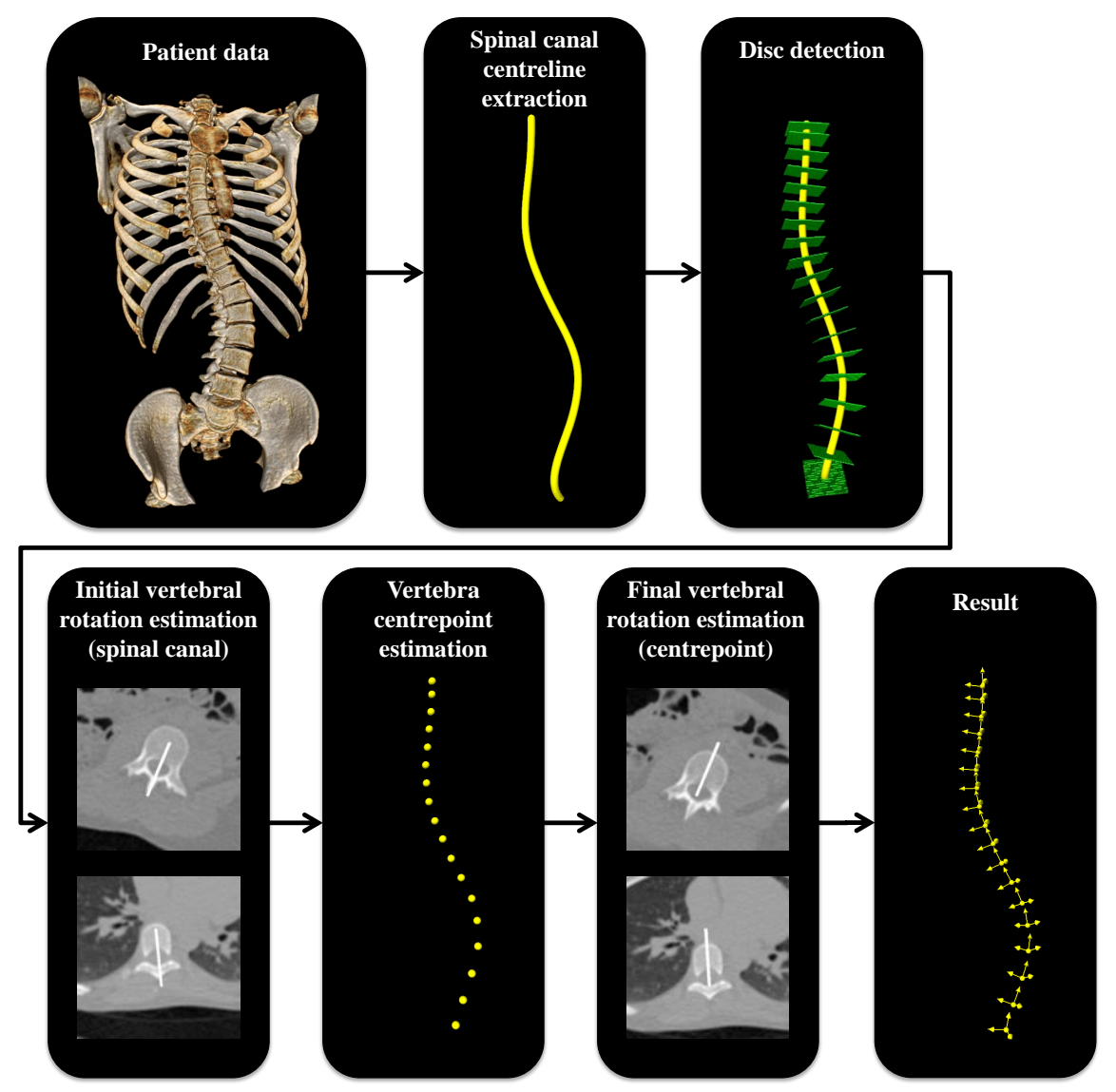

Figure 3. Schematic overview of the pre-processing employed for estimating an initial position and rotation of each vertebra.

where $B$ is a $3 \mathrm{D}$ window mask, in our case a $3 \times 3 \times 3 \mathrm{~mm}^{3}$ mask, $\oplus$ denotes dilation and $\ominus$ denotes erosion, i.e. the valley image $V(X)$ is constructed by subtracting the original image from its morphologically closed counterpart and the valley-emphasized image is obtained by subtracting the valley image from the original image. The result of this operation is illustrated in figure 4

\section{Model-based spine registration}

Given the approximate pose of each vertebra, the spine model is then registered to the spine of the patient, vertebra by vertebra, first with an affine registration followed by a deformable registration. The vertebrae were registered in the order L5 to T1. The reason for applying this sequential registration process is two-fold. Firstly, it provides a more robust registration than using only a deformable registration, since the applied regularization in deformable registration has a bias to penalize affine transformations unless employing curvature regularization as proposed by Fischer and Modersitzki (2003). Secondly, the registration of sub-volumes has the advantage of allowing the use of graphics processing units (GPUs) for improved computational performance. 


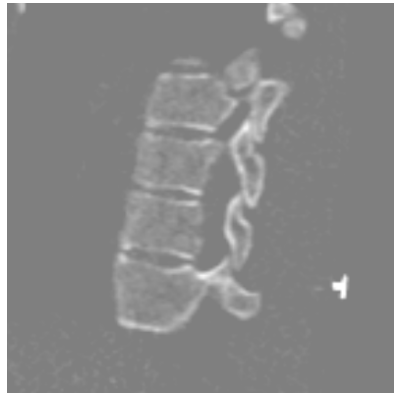

(a)

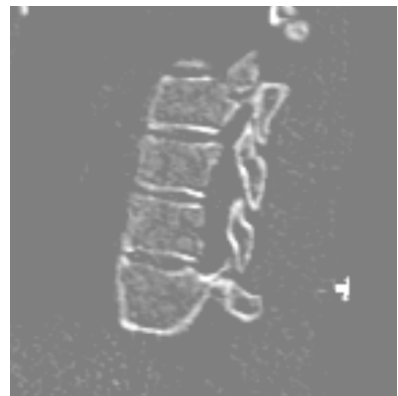

(b)

Figure 4. The applied valley-emphasis was needed since the processed data consisted of low-dose CT data, where the separating structures between surrounding vertebrae were not always clearly distinguishable. Especially the vertebral process were difficult to distinguish for the thoracic vertebrae. For example, compare (a) with (b), before respectively after valley-emphasis.

The GPU is typically not applicable to use when working with large data volumes, which is due to the current limitations regarding the amount of memory available on the GPUs, causing many GPU-based implementations of registration algorithms to be limited to data sets of the size $256 \times 256 \times 256$ or smaller, (Han et al. 2009, Gu et al. 2010).

In this work, we have decided to employ image registration based upon phasedifference. This is due to the fact that the local phase of a signal is invariant to signal energy and provides sub-pixel accuracy by varying smoothly. Especially the first reason, invariance to signal energy, is relevant in the case of model-based registration, since the signal intensities of the spine model and the patient data are

not likely to match. Hence, the use of simple similarity measures, e.g. the sum of squared intensity differences, are unlikely to perform well in this scenario. In addition, the use of phase-difference is more attractive then using more elaborate measures, e.g. mutual information, since they come with an additional computational cost. The local phase of an image can be estimated using oriented quadrature filters (Granlund and Knutsson 1995). The initial affine registration is done employing the algorithm described by Hemmendorff et al. (2002) and implemented on the GPU using the compute unified device architecture (CUDA) by Eklund et al. (2010). The final deformable registration uses the registration algorithm known as the Morphon. This method was introduced by Knutsson and Andersson (2005) and implemented in CUDA by Forsberg et al. (2011). The following subsections provide a brief introduction to phase-based image registration (affine and deformable).

\subsection{Affine registration}

The affine phase-based registration is based upon the traditional optical flow equation

$$
\nabla I^{T} \mathbf{v}-\Delta I=0
$$

but replaced with its phase-based counterpart

$$
\nabla \varphi_{k}^{T} \mathbf{v}-\Delta \varphi_{k}=0
$$


where

$$
\begin{aligned}
\Delta \varphi_{k} & =\varphi_{1}-\varphi_{2}=\arg \left(q_{1}, q_{2}^{*}\right), \\
q_{1, k} & =I_{1} * f_{k}, \\
q_{2, k} & =I_{2} * f_{k},
\end{aligned}
$$

$f_{k}$ is a quadrature filter in the spatial domain with the orientation $\hat{\mathbf{n}}_{k}$, and $I_{1}$ and $I_{2}$ are the two images to be registered. The displacement $\mathbf{v}$ is estimated by utilizing a parametrization of the displacement field

$$
\mathbf{v}(\mathbf{x})=\left[\begin{array}{lll}
p_{1} & p_{2} & p_{3} \\
p_{4} & p_{5} & p_{6} \\
p_{7} & p_{8} & p_{9}
\end{array}\right]\left[\begin{array}{l}
x \\
y \\
z
\end{array}\right]+\left[\begin{array}{l}
p_{10} \\
p_{11} \\
p_{12}
\end{array}\right]=B(\mathbf{x}) \mathbf{p}
$$

where

$$
\begin{aligned}
B(\mathbf{x}) & =\left[\begin{array}{llllllllllll}
x & y & z & 0 & 0 & 0 & 0 & 0 & 0 & 1 & 0 & 0 \\
0 & 0 & 0 & x & y & z & 0 & 0 & 0 & 0 & 1 & 0 \\
0 & 0 & 0 & 0 & 0 & 0 & x & y & z & 0 & 0 & 1
\end{array}\right], \\
\mathbf{p} & =\left[p_{1}, p_{2}, p_{3}, p_{4}, p_{5}, p_{6}, p_{7}, p_{8}, p_{9}, p_{10}, p_{11}, p_{12}\right]^{T},
\end{aligned}
$$

and defining an $L_{2}$ norm,

$$
\epsilon^{2}=\frac{1}{2} \sum_{k} \sum_{i} c_{i k}\left[\nabla \varphi_{k}\left(\mathbf{x}_{i}\right)^{T} B\left(\mathbf{x}_{i}\right) \mathbf{p}-\Delta \varphi_{k}\left(\mathbf{x}_{i}\right)\right]^{2},
$$

where

$$
c_{k}=\sqrt{\left|q_{1 k} q_{2 k}\right|} \cos ^{2}\left(\frac{\Delta \varphi_{k}}{2}\right) .
$$

Differentiating $\epsilon^{2}$ with respect to $\mathbf{p}$ and setting to 0 gives

$$
\underbrace{\sum_{k} \sum_{i} c_{i k} B_{i}^{T} \nabla \varphi_{i k} \nabla \varphi_{i k}^{T} B_{i}}_{A} \mathbf{p}=\underbrace{\sum_{k} \sum_{i} c_{i k} B_{i}^{T} \nabla \varphi_{i k} \Delta \varphi_{i k}}_{\mathbf{h}},
$$

and the solution

$$
\mathbf{p}=A^{-1} \mathbf{h} .
$$

Variables in 15) with the subscript $i$ corresponds to the values at the different spatial locations $\mathbf{x}_{i}$, i.e. $B_{i}=B\left(\mathbf{x}_{i}\right)$. The described algorithm is easily applied in an iterative multi-resolution framework by accumulating $\mathbf{p}$, setting $\tilde{I}_{2}(\mathbf{x})=I_{2}((\mathbf{x})+\mathbf{v}(\mathbf{x}))$ and replacing $I_{2}$ with $\tilde{I}_{2}$ in 9 .

\subsection{Deformable registration}

The deformable phase-based registration algorithm, i.e. the Morphon introduced in (Knutsson and Andersson 2005), can be embedded into a generic registration framework for non-parametric image registration with decoupled similarity optimization and regularization (Janssens et al. 2011) as follows (using the same notation as in the previous section).

Let $\mathbf{d}_{u}$ denote the update field and $\mathbf{d}$ the total displacement field. Define a pixel-wise least squares problem as

$$
\epsilon^{2}=\sum_{k}\left[c_{k} \mathbf{T}\left(d_{k} \hat{\mathbf{n}}_{k}-\mathbf{d}_{u}\right)\right]^{2},
$$


where $\mathbf{T}$ is the local structure tensor, $d_{k}$ the displacement in direction $k$ associated with the phase-difference according to $d_{k}=\Delta \varphi_{k} / \rho$ ( $\rho$ is the centre frequency of the applied quadrature filter), and $c_{k}$ as previously defined. Differentiating and setting the derivatives to 0 will provide a linear equation system, which is easily solved, providing a pixel-wise update field.

$$
A \mathbf{d}_{u}=\mathbf{b} \quad \Leftrightarrow \quad \mathbf{d}_{u}=A^{-1} \mathbf{b}
$$

For fluid-like registration, regularize $\mathbf{d}_{u}$ with a Gaussian kernel

$$
\mathbf{d}_{u}=\mathbf{d}_{u} * g_{\text {fluid }}
$$

and then accumulate the update field to the total displacement field

$$
\mathbf{d}=\mathbf{d}+\mathbf{d}_{u} .
$$

Finally, if diffusion-like registration, regularize $\mathbf{d}$ with a Gaussian kernel

$$
\mathbf{d}=\mathbf{d} * g_{\text {diff }} .
$$

In this work, the estimated transformation is used to transfer the landmarks of the model to the patient data. Hence, it is important to have an effective way of computing the inverse transform. Since the Morphon is embedded into the generic registration framework, which is similar to the traditional framework of the demons registration algorithm (Thirion 1998), the approach described in (Vercauteren et al. 2008) is applicable for implementing a symmetric registration based upon diffeomorphic registration in the log-domain. This is achieved using stationary velocity fields for encoding the spatial transformation. Apart from providing a symmetric registration, this approach also has the benefit of providing an simple method for computing the inverse transformation. Details of this are omitted for the sake of space but can be found in (Vercauteren et al. 2008).

\subsection{Registration Parameters}

The following parameters were used during the registration process:

- Extracted sub-volume size $-128 \times 128 \times 128$ voxels for the lumbar vertebrae and $96 \times 96 \times 96$ voxels for the thoracic vertebra.

- Number of scales - For the affine registration we employed two scales corresponding to a resampling of a factor two and four, and for the deformable registration we employed three scales corresponding to the original scale and to a resampling of a factor two and four.

- Number of iterations per scale - A fixed number of iterations was employed, corresponding to ten iterations per scale except for the original scale when only five iterations were employed.

- Regularization - Both fluid-like and diffusion-like registration was employed, using a Gaussian kernel with $\sigma$ set to 2.5 pixels.

\section{Evaluation}

In order to evaluate the proposed method, four $\mathrm{CT}$ data sets containing the spines from patients suffering from scoliosis were retrospectively gathered from the picture archiving and communication system at the local hospital. All data sets included vertebrae L5-T1, i.e. 17 vertebrae each and 68 vertebrae in total. The patients were 
$15 \pm 1.7$ years old at the time of the examination with an Cobb angle of $62.9^{\circ} \pm 17.0$, as measured on standing anterior-posterior radiographs. The images were captured as a part of the standard routine for pre-operational planning and a specific lowdose protocol was in use, in order to minimize the delivered radiation dose (Kalra et al. 2013). Before processing, the image data was resampled to an isotropic resolution of $1 \times 1 \times 1 \mathrm{~mm}^{3}$ using tri-linear interpolation, in order to allow the usage of quadrature filters with isotropic resolution. All datasets had before the resampling a voxel size of less than $1 \times 1 \times 1 \mathrm{~mm}^{3}$. Note that this data is the same data as employed in (Forsberg et al. 2013).

Three different measures were employed to evaluate the proposed method, two measures evaluating the segmentation/registration accuracy, and one measure for evaluating the actual estimates derived from the transferred landmarks. As can be noted, the emphasis in the evaluation is on registration accuracy, which is natural, since the method is based on image registration. For the sake of evaluating the registration accuracy, each vertebra (in all four patients) were marked with the corresponding landmarks as defined for the model in section 2.2. In addition, each vertebra was manually segmented. Thereafter, each dataset was processed according to the proposed method. The manually placed landmarks and the transferred landmarks were used to assess the registration accuracy by employing the target registration error (TRE), i.e. the distance between corresponding landmarks. The manual hand segmentations were compared with the segmentations obtained form the deformed models using a point-to-surface error (PSE). The PSE is defined as the smallest distance between a node on the deformed model and the closest surface of the manual segmentation. The meshes defining the vertebrae of the deformed model and the hand-segmented vertebrae were acquired from their corresponding binary volumes using iso2mesh (Fang and Boas 2009). The surface of each vertebra was approximated using 1500-3500 nodes, where the number of nodes depended on the actual size of the vertebra.

To evaluate the estimated measures from the transferred landmarks, the AVR for all vertebrae were manually measured by three observers employing the method of Aaro and Dahlborn (1981). This is done by measuring the angle between a straight line through the dorsal central aspect of the vertebral foramen and the centrepoint of the vertebral body, and a line parallel to the sagittal image plane of the image volume. To compensate for the possible tilt of the vertebral body in the sagittal and/or the coronal plane, the image data was resampled using curved planar reformation. The resampling was done orthogonal to a curve running through the centrepoints of the vertebral bodies, centrepoints defined by each observer. The average AVR measurement from the three observers for each vertebrae was considered as the ground truth AVR. Corresponding AVR measurements are easily derived from the estimated rotation matrices $\mathbf{R}$ of each vertebra. The AVR is estimated as the angle between the normal vector of the coronal plane of the vertebra and the normal vector of the coronal plane of the image volume, projected onto the axial plane of the vertebra. Mean and standard deviation of the absolute difference in AVR measurements (MAD) between manual and automatic measurements, were used to evaluate the AVR measurements.

All three measures were estimated for using only affine registration (A) and for using affine registration and deformable registration $(A+D)$. Results from the evaluation are given in table 1 . 
Table 1. Validation results comparing transferred landmarks with manually placed landmarks, model-based segmentation with manual segmentation, and automatically measured AVR with manually measured AVR.

\begin{tabular}{cccc}
\hline & TRE $[\mathrm{mm}]$ & PSE $[\mathrm{mm}]$ & $\Delta$ MAD $\left[^{\circ}\right]$ \\
\hline $\mathrm{A}^{\mathrm{a}}$ & $3.0 \pm 1.8$ & $1.3 \pm 1.0$ & $2.8 \pm 1.9$ \\
$\mathrm{~A}+\mathrm{D}^{\mathrm{b}}$ & $2.3 \pm 1.7$ & $0.9 \pm 0.9$ & $2.5 \pm 1.8$ \\
\hline
\end{tabular}

a Affine registration

b Deformable registration

\section{Discussion}

In this paper, a method has been presented, based upon fitting a spine model via image registration and the transfer of landmarks to patient data, for segmenting the vertebrae and for estimating position and rotation of each vertebra in the spine. This is of great relevance for several diseases causing a deformation of the spinal curvature and/or the vertebrae themselves. In this work, we have focused on idiopathic scoliosis, a disease in need of better methods and measures for automatically quantifying and assessing induced spinal deformities. We believe that the proposed method can be a useful tool for clinicians in their research related to idiopathic scoliosis, to explore underlying mechanisms of the disease, and eventually in their daily clinical work for determining the most suitable treatment for individual patients.

The proposed method has several advantages compared to other computerized methods for assessing spinal deformities, where one of the strongest advantages is combining an accurate segmentation of the vertebrae with reliable estimates of various measures related to spinal deformities. Compared to methods assessing rotation (mainly AVR) our method differs by not relying on the symmetry of the vertebral body for measuring the rotation of the vertebral body, which is considered as relevant since the vertebral body often can be severely deformed due to the scoliosis. Note, though, that this aspect could not be fully explored in this paper, since the included data sets lacked severely deformed vertebral bodies. In addition, the method is fully automatic, thereby reducing any observer variability. Further, the method benefits from the extensive usage of the GPU for executing various computations, providing a computation time of about three to four minutes to process all 17 lumbar and thoracic vertebrae in an image volume, given the current implementation and a data size of approximately $300 \times 300 \times 700$ voxels.

Compared to stereo-radiography and 3D reconstruction based upon the EOS $^{\circledR}$ system, our proposed method has the benefit of not requiring a dedicated imaging modality but can be used with standard CT. However, despite efforts to reduce dose in spinal CT examinations for children and adolescents (Kalra et al. 2013), the $\operatorname{EOS}^{\circledR}$ system based upon stereo-radiography and 3D reconstruction is still likely to deliver a significantly lower dose. Note that the study by Kalra et al. (2013) showed that the radiation dose for standard radiography is only two to three times lower than using low dose CT.

Regarding the results provided from the evaluation and presented in table 1 , there are a number of things to comment on. First of all, it can be noted that the difference between using affine only and affine + deformable registration is not that large. This is likely due to, as already mentioned, that the included patients did not have severely deformed vertebra, although, in general, the use of affine + deformable registration 
provides better results.

Furthermore, the average PSE $0.9 \mathrm{~mm} \pm 0.9$ suggests a very good fit of the deformed spine model. In fact, the PSE is slightly better than the results reported in (Klinder et al. 2009, Štern et al. 2011), $1.12 \mathrm{~mm} \pm 1.04$ respectively $1.17 \mathrm{~mm} \pm 0.33$, two fully automatic methods for segmenting the vertebrae. Noteworthy in this context is also that both Klinder et al. (2009) and Štern et al. (2011) report rather extensive computation times, close to 40 minutes for 12 vertebrae in (Klinder et al. 2009) and approximately 1 to 15 minutes per vertebra in (Štern et al. 2011), which can be compared with our computation time of three to four minutes for 17 vertebrae. The relevance of this comparison can be questioned, since both Klinder et al. (2009) and Štern et al. (2011) implement their methods on the CPU and not on the GPU as in our work. However, determining whether it would be possible to improve the computation time of their methods by utilizing the GPU is outside the scope of this work, since not all image processing methods are suitable for parallel processing on the GPU. Regarding the results in (Štern et al. 2011), it should also be pointed out that they only estimate the PSE for a limited number of landmarks attached only to the vertebral bodies and that their reported results only contain the successful cases, i.e. where the PSE was less than $3 \mathrm{~mm}$. An advantage of the method in (Klinder et al. 2009) is that their method provides automatic identification of the vertebrae as well. Note that given the segmentations of the vertebrae as provided by the methods of Klinder et al. (2009) and Štern et al. (2011), these methods could probably also be utilized to derive measures such as position and rotation of each vertebra. Despite the low PSE of our method, it should be noted that the errors are in general larger for the processes of the vertebrae, depicted in figure 5, although the imperfect registration of the transverse processes appear to not affect the PSE in general. In addition, the thoracic vertebrae have in general a larger error, e.g. the lumbar vertebrae have an average PSE of $0.7-0.8 \mathrm{~mm}$ whereas the average PSE of the thoracic vertebrae ranges from $0.7 \mathrm{~mm}$ to $1.3 \mathrm{~mm}$.

Comparing transferred landmarks and manually placed landmarks provides an average TRE of $2.3 \mathrm{~mm} \pm 1.7$. This is slightly better than the results $(3.3 \mathrm{~mm} \pm 3.8)$ reported by Delorme et al. (2003) for evaluating the accuracy of using stereoradiography and reconstruction techniques for 3D modeling of the vertebrae. Here it is interesting to note the difference between the PSE and the TRE, since the PSE suggests a much better registration accuracy than the TRE. The difference is believed to be due to the fact that corresponding landmarks are in general difficult to place in corresponding anatomical positions. In this work, the situation is even worse since one set of the landmarks are placed in patient data and the other set on a model, e.g. a perfect one-to-one mapping between the model and the patient cannot be expected. For example, the intra-observer variability for placing the landmarks in the model was $1.4 \mathrm{~mm} \pm 0.7$. Hence, we consider the obtained TRE together with the PSE as sufficient for suggesting that a decent registration accuracy has been achieved. The results of the registration accuracy can be further observed in figure 6 .

Comparing the AVR as estimated from transferred landmarks with corresponding manual measurements of the same, provides an MAD of $2.5^{\circ} \pm 1.8$. This result can be compared with results reported in (Vrtovec et al. 2010) and results obtained by employing the method in (Forsberg et al. 2013), i.e. the pre-processing step of our proposed method, corresponding to $1.0^{\circ} \pm 1.3$ and $2.5^{\circ} \pm 2.6$ respectively. Hence, the difference in AVR measurements of the proposed method, when comparing computerized and manual measurements, is roughly on par with earlier reported 


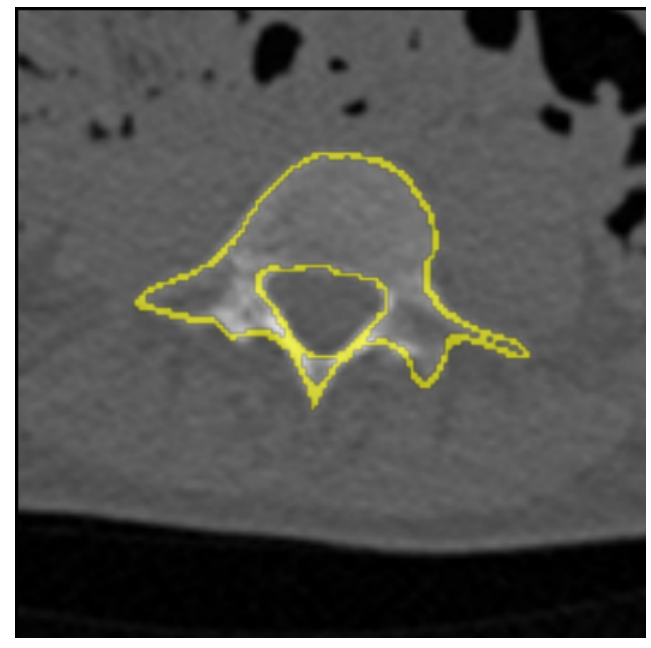

(a)

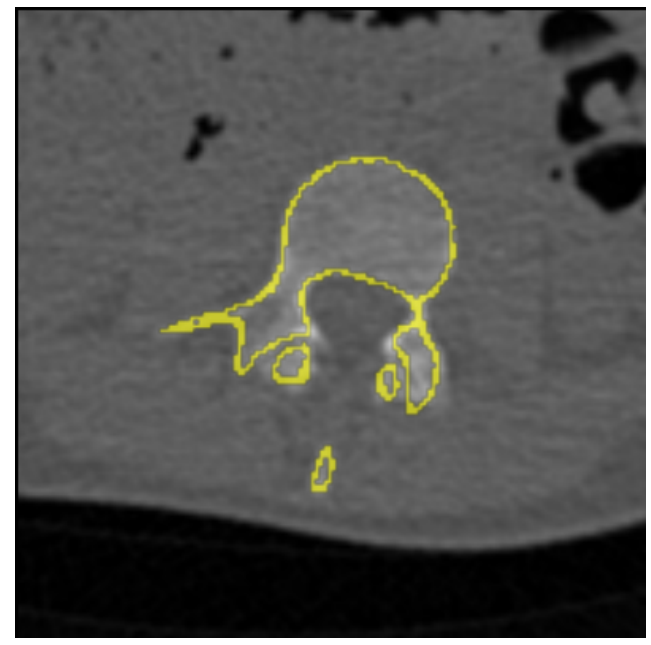

(c)

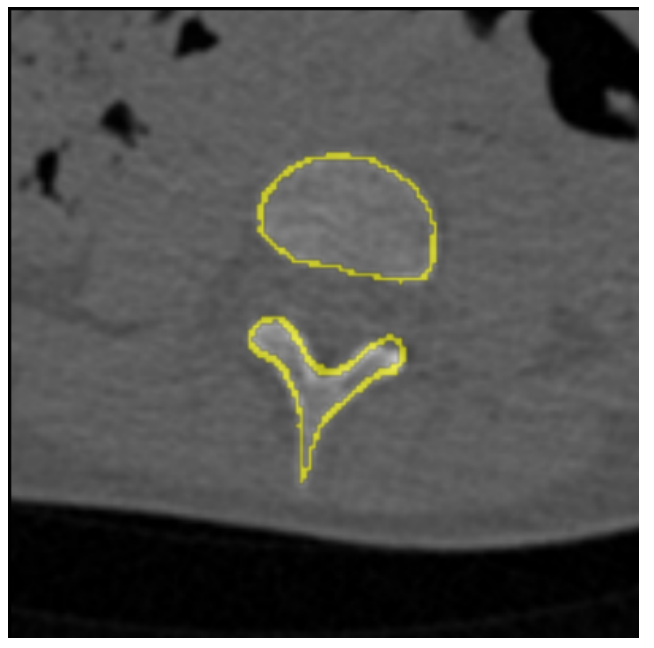

(b)

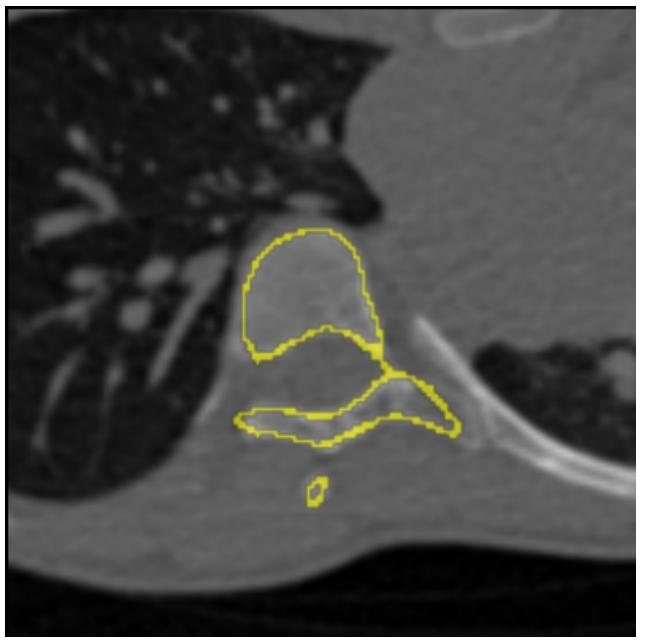

(d)

Figure 5. The registration accuracy is in general excellent for the vertebral bodies, as given by (a)-(d). However, the registration accuracy decreases especially for the superior articular processes (c) and the transverse processes (d), where the latter are sometimes difficult to distinguish from the ribs. In general, the registration accuracy decreases for the thoracic vertebrae.

results. However, the comparison with the results in (Vrtovec et al. 2010) is questionable, since the evaluation was based on MRI data using only two patients, one scoliotic (mild scoliosis corresponding to a Cobb angle of $14^{\circ}$ ), and not including all lumbar and thoracic vertebrae. Furthermore, the obtained difference can be compared with the inter-observer difference, which was $1.9^{\circ} \pm 1.5$ (MAD) for the observers included in this evaluation, and the difference obtained when employing the manually placed landmarks on the patient data, which corresponds to $1.9^{\circ} \pm 1.7$ (MAD).

Since the results reported in (Forsberg et al. 2013) are based upon 


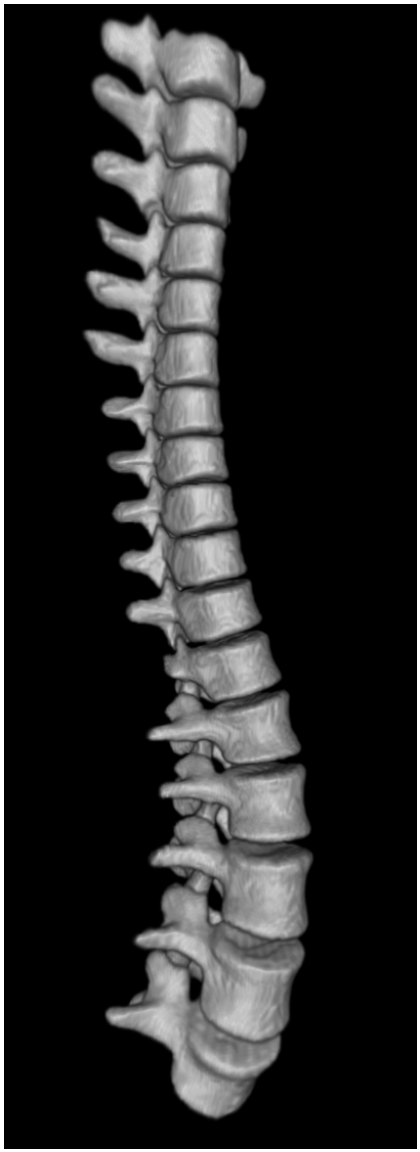

(a)

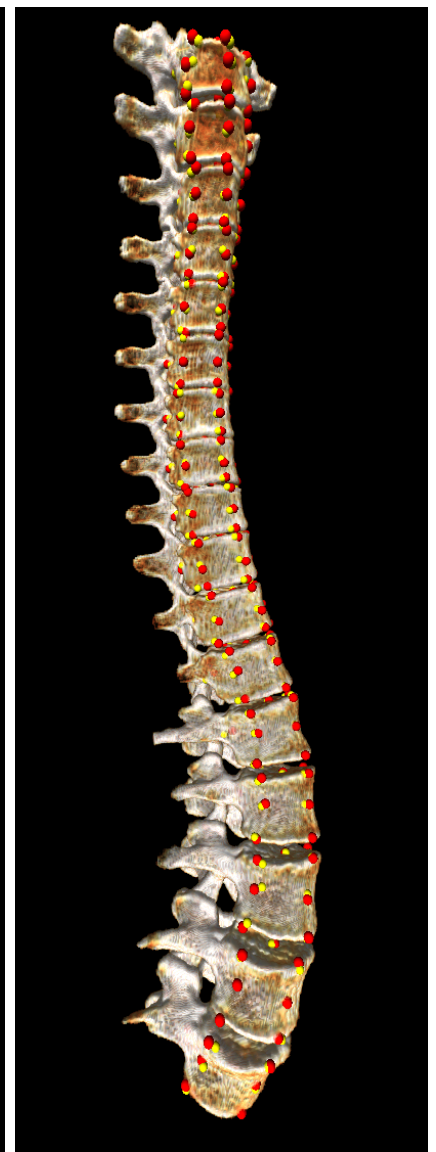

(b)

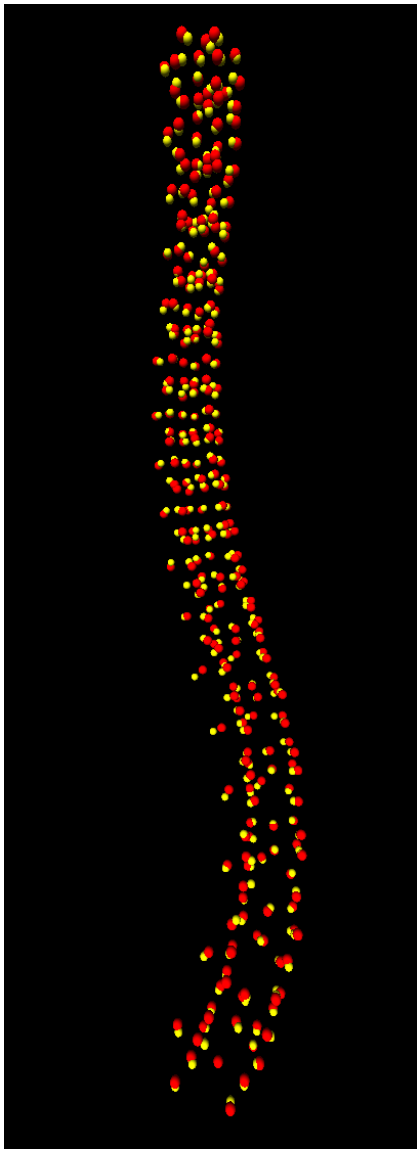

(c)

Figure 6. The deformed model is visualized in (a) and where (b) shows the patient data. In both (b) and (c) the transferred landmarks (in red) and the manually placed landmarks (yellow) can be observed.

the same data set as used in this work, the following remarks regarding the results of the proposed method and of the method used in (Forsberg et al. 2013) are relevant. First, it can be noted that the correlation between the two sets of measurements corresponds to 0.965 , which is similar to the correlation between the obtained measurements and the manual measurements, corresponding to 0.967 and 0.961 (the former refers to the measurements obtained with the proposed method). Second, plotting the difference reveals no appearent systematic differences between the two sets of measurements. Hence, for these data sets the two methods appear to provide measurements that have similar accuracy and with no appearent systematic difference between them. However, note that the method in (Forsberg et al. 2013) is limited to only measure the rotation of a vertebra and relies on the symmetry of the vertebra for this estimation, whereas the proposed method can provide any type of measure, given 
that these measures can be estimated based upon a set of well-defined landmkars, and is not limited to symmetrical vertebrae. In addition, the proposed method provides accurate segmentations of the vertebrae.

Apart from the evaluation results, there are a few other things that need to be commented on in regards to the proposed method. In this work, we have focused on evaluating the registration accuracy and the accuracy of the AVR measurements when compared to manual measurements. This evaluation should be extended to a larger set of patients and to also include other measures derived from the landmarks. Especially measures assessing the deformity of the vertebral bodies should be pursued, in order to provide tools for assessing the vertebral deformation during the progression of scoliosis. In addition, there is also more work to be done in terms of defining how the rotation measurements should be estimated. Another aspect that would be interesting to pursue further is to replace the valley-emphasis, applied in the pre-processing, with some other method based on adaptive filtering, which can provide a much stronger enhancement of the contained structures. In this case, it is not necessary to consider visual aspects of the enhancement of the image data, which allows a very strong edge enhancement to be applied. This could potentially improve the registration accuracy for the processes of the vertebrae.

In all, the proposed method shows promising results in terms of providing an automatic method applicable to CT data for estimating position and rotation of each vertebrae in the spine. Especially the fact that the method is not limited to the current set of landmarks for the different measurements, makes it interesting and adaptable. In addition, the method shows great potential in being used for segmentation of the vertebrae in $\mathrm{CT}$ data.

\section{Acknowledgments}

The authors would like to thank L. Vavruch and H. Tropp at the Department of Clinical and Experimental Medicine, Linköping University, Sweden, for valuable input in discussions regarding idiopathic scoliosis and for assistance in collecting the used image data. This work was funded by the Swedish Research Council (grant 2007-4786) and the Swedish Foundation for Strategic Research (grant SM10-0022). Visualization and manual measurements were performed using MeVisLab (provided by Fraunhofer MEVIS.

\section{References}

Aaro, S. and Dahlborn, M.: 1981, Estimation of vertebral rotation and the spinal and rib cage deformity in scoliosis by computer tomography, Spine 6(5), 460-467.

Adam, C. J. and Askin, G. N.: 2006, Automatic measurements of vertebral rotation in idiopathic scoliosis, Spine 31(3), E80-E83.

Brown, R. H., Burstein, A. H., Nash, C. L. and Schock, C. C.: 1976, Spinal analysis using a threedimensional radiographic technique, Journal of Biomechanics 9(6), 355-IN1.

Cobb, R. J.: 1948, Outline for study of scoliosis, American Academy of Orthopaedic Surgeons, Instructional Course Lectures 5, 261-275.

Delorme, S., Petit, Y., De Guise, J. A., Labelle, H., Aubin, C.-E. and Dansereau, J.: 2003, Assessment of the 3-D reconstruction and high-resolution geometrical modeling of the human skeletal trunk from 2-D radiographic images, Biomedical Engineering, IEEE Transactions on $\mathbf{5 0}(8), 989-998$.

Dubousset, J., Charpak, G., Dorion, I., Skalli, W., Lavaste, F., Deguise, J., Kalifa, G. and Ferey, S.: 2005, A new 2D and 3D imaging approach to musculoskeletal physiology and pathology 
with low-dose radiation and the standing position: the EOS system, Bulletin de l'Academie nationale de medecine 189(2), 287-297.

Eklund, A., Andersson, M. and Knutsson, H.: 2010, Phase based volume registration using CUDA, Acoustics Speech and Signal Processing (ICASSP), 2010 IEEE International Conference on, IEEE, pp. 658-661.

Fang, Q. and Boas, D. A.: 2009, Tetrahedral mesh generation from volumetric binary and grayscale images, Biomedical Imaging: From Nano to Macro, 2009. ISBI'09. IEEE International Symposium on, IEEE, pp. 1142-1145.

Fischer, B. and Modersitzki, J.: 2003, Curvature based image registration, Journal of Mathematical Imaging and Vision 18(1), 81-85.

Forsberg, D., Eklund, A., Andersson, M. and Knutsson, H.: 2011, Phase-based non-rigid 3D image registration - from minutes to seconds using CUDA, HP-MICCAI / MICCAI-DCI 2011.

Forsberg, D., Lundström, C., Andersson, M., Vavruch, L., Tropp, H. and Knutsson, H.: 2013, Fully automatic measurements of axial vertebral rotation for assessment of spinal deformity in idiopathic scoliosis, Physics in Medicine and Biology 58(6), 1775-1787.

Granlund, G. and Knutsson, H. (eds): 1995, Signal Processing for Computer Vision, Kluwer Academic Publishers.

Gu, X., Pan, H., Liang, Y., Castillo, R., Yang, D., Choi, D., Castillo, E., Majumdar, A., Guerrero, T. and Jiang, S. B.: 2010, Implementation and evaluation of various demons deformable image registration algorithms on a GPU, Physics in Medicine and Biology 55(1), 207-219.

Han, X., Hibbard, L. S. and Willcut, V.: 2009, GPU-accelerated, gradient-free MI deformable registration for atlas-based MR brain image segmentation, Computer Vision and Pattern Recognition Workshops, 2009. CVPR Workshops 2009. IEEE Computer Society Conference on, IEEE, pp. 141-148.

Hawes, M. C. and O'Brien, J. P.: 2006, The transformation of spinal curvature into spinal deformity: pathological processes and implications for treatment, Scoliosis 1(1), 3.

Heidari, B., Fitzpatrick, D., McCormack, D. and Synnott, K.: 2006, Correlation of an induced rotation model with the clinical categorization of scoliotic deformity - a possible platform for prediction of scoliosis progression, Studies in Health Technology and Informatics 123, 169175 .

Hemmendorff, M., Andersson, M., Kronander, T. and Knutsson, H.: 2002, Phase-based multidimensional VOLUME registration, IEEE Transactions on Medical Imaging 21(12), 1536-1543.

Janssens, G., Jacques, L., de Xivry, J., Geets, X. and Macq, B.: 2011, Diffeomorphic registration of images with variable contrast enhancement, Journal of Biomedical Imaging p. 16.

Kalra, M. K., Quick, P., Singh, S., Sandborg, M. and Persson, A.: 2013, Whole spine CT for evaluation of scoliosis in children: feasibility of sub-millisievert scanning protocol, Acta Radiologica 54(2), 226-230.

Kim, Y. and Kim, D.: 2009, A fully automatic vertebra segmentation method using 3D deformable fences, Computerized Medical Imaging and Graphics 33(5), 343-352.

Klinder, T., Ostermann, J., Ehm, M., Franz, A., Kneser, R. and Lorenz, C.: 2009, Automated model-based vertebra detection, identification, and segmentation in CT images., Medical Image Analysis 13(3), 471-482.

Knutsson, H. and Andersson, M.: 2005, Morphons: Segmentation using elastic canvas and paint on priors, Image Processing (ICIP), 2005 IEEE International Conference on, IEEE, pp. II$1226-9$.

Kouwenhoven, J. W. M., Vincken, K. L., Bartels, L. W. and Castelein, R. M.: 2006, Analysis of pre-existent vertebral rotation in the normal spine, Spine 31(13), 1467-1472.

Kuklo, T., Potter, B. K. and Lawrence, L.: 2005, Vertebral rotation and thoracic torsion in adolescent idiopathic scoliosis: What is the best radiographic correlate?, Journal of Spinal Disorders and Techniques 18(2), 139-147.

Lam, G., Hill, D., Le, L., Raso, J. and Lou, E.: 2008, Vertebral rotation measurement: a summary and comparison of common radiographic and CT methods, Scoliosis 3(1), 16.

Moura, D. C., Boisvert, J., Barbosa, J. G., Labelle, H. and Tavares, J. M. R.: 2011, Fast 3D reconstruction of the spine from biplanar radiographs using a deformable articulated model, Medical Engineering \&6 Physics 33(8), 924-933.

Rogers, B. P., Haughton, V. M., Arfanakis, K. and Meyerand, M. E.: 2002, Application of image registration to measurement of intervertebral rotation in the lumbar spine, Magnetic Resonance in Medicine 48(6), 1072-1075.

Segars, W. P., Sturgeon, G., Mendonca, S., Grimes, J. and Tsui, B. M.: 2010, 4D XCAT phantom for multimodality imaging research, Medical Physics 37(9), 4902-4915.

Skalli, W., Lavaste, F. and Descrimes, J.: 1995, Quantification of three-dimensional vertebral 
rotations in scoliosis: what are the true values?, Spine 20(5), 546-553.

Štern, D., Likar, B., Pernuš, F. and Vrtovec, T.: 2011, Parametric modelling and segmentation of vertebral bodies in 3D CT and MR spine images, Physics in Medicine and Biology 56(23), 7505-7522.

Thirion, J. P.: 1998, Image matching as a diffusion process: an analogy with Maxwell's demons, Medical Image Analysis 2(3), 243-260.

Vercauteren, T., Pennec, X., Perchant, A. and Ayache, N.: 2008, Symmetric log-domain diffeomorphic registration: A demons-based approach, Medical Image Computing and Computer-Assisted Intervention MICCAI 2008, Vol. 5241 of Lecture Notes in Computer Science, Springer, pp. $754-761$.

Villemure, I., Aubin, C. E., Grimard, G., Dansereau, J. and Labelle, H.: 2001, Progression of vertebral and spinal three-dimensional deformities in adolescent idiopathic scoliosis: a longitudinal study, Spine 26(20), 2244-2250.

Vrtovec, T.: 2008, Modality-independent determination of vertebral position and rotation in 3D, Medical Imaging and Augmented Reality, Vol. 5128 of Lecture Notes in Computer Science, Springer, pp. 89-97.

Vrtovec, T., Pernuš, F. and Likar, B.: 2009, A review of methods for quantitative evaluation of spinal curvature, European Spine Journal 18(5), 593-607.

Vrtovec, T., Pernuš, F. and Likar, B.: 2009, A review of methods for quantitative evaluation of axial vertebral rotation, European Spine Journal 18, 1079-1090.

Vrtovec, T., Pernuš, F. and Likar, B.: 2010, Determination of axial vertebral rotation in MR images: comparison of four manual and a computerized method, European Spine Journal 19, 774-781. 